(C) 1997 International Press

Adv. Theor. Math. Phys. 1 (1997) 115-126

\title{
Dynamics of Multiple Kaluza-Klein Monopoles in M-Theory and String Theory
}

\author{
Ashoke Sen ${ }^{1}$ \\ Mehta Research Institute of \\ Mathematics and Mathematical Physics \\ Chhatnag Road, Jhusi, Allahabad 221506, INDIA
}

\begin{abstract}
We analyse the world-volume theory of multiple Kaluza-Klein monopoles in string and M-theory by identifying the appropriate zero modes of various fields. The results are consistent with supersymmetry, and all conjectured duality symmetries. In particular for M-theory and type IIA string theory, the low energy dynamics of $N$ Kaluza-Klein monopoles is described by supersymmetric $U(N)$ gauge theory, and for type IIB string theory, the low energy dynamics is described by a $(2,0)$ supersymmetric field theory in $(5+1)$ dimensions with $N$ tensor multiplets and tensionless self-dual strings. It is also argued that for the Kaluza-Klein monopoles in heterotic string theory, the apparently flat moduli space gets converted to the moduli space of BPS monopoles in $S U(2)$ gauge theory when higher derivative corrections to the supergravity equations of motion are taken into account.
\end{abstract}

\section{Introduction}

Among the brane solutions in M-theory and various string theories are Kaluza-Klein monopoles [1,2], which represent six-branes in M-theory compactified on a circle, and five-branes in various string theories compactified on

\footnotetext{
${ }^{1}$ On leave of absence from Tata Institute of Fundamental Research, Homi Bhabha Road, Bombay 400005, INDIA.
} 
a circle. Various conjectured string dualities relate these solitons to various other known solitons in string theory. For example, in type IIA (IIB) string theory, T-duality relates these to appropriate solitonic five-branes in type IIB (IIA) string theory, whereas in type IIA string theory and $M$-theory, U-duality relates the Kaluza-Klein monopoles to appropriate D-branes in type IIA string theory. In this paper we shall explicitly test these duality conjectures by comparing the low energy dynamics of multiple Kaluza-Klein monopoles to that of various other string solitons. Dynamics of KaluzaKlein monopoles have been analysed previously in different contexts in references [3-7].

Kaluza-Klein monopole solutions $[1,2]$ have the special property that at a generic point in the moduli space they represent completely non-singular solutions of the low energy supergravity equations of motion. The curvature can be kept small everywhere by taking the radius of the compact direction and the distance between different monopoles sufficiently large, whereas the string coupling can be kept small everywhere by keeping the asymptotic coupling constant small. This means that, at least at a generic point in the moduli space, we can reliably analyse the low energy dynamics of these monopoles using the weak coupling, low energy supergravity equations of motion. At special points in the moduli space, when two or more monopoles coincide, stringy effects become important. As we shall see, in this case the dynamics can be approximated by string propagation in an appropriate ALE space, - a problem that has already been studied in other contexts. We shall find that the dynamics of the Kaluza-Klein monopoles, both at generic and at special points in the moduli space, are consistent with various duality conjectures.

Besides providing new tests of various duality conjectures, the world volume theory of Kaluza-Klein monopoles can also be used to study novel supersymmetric theories. For example, for type IIB string theory, the world volume theory of $N$ Kaluza-Klein monopoles is a $(5+1)$ dimensional theory with $(2,0)$ supersymmetry and $N$ tensor multiplets, and also contains selfdual strings which become tensionless when the locations of some of the monopoles coincide. This is closely related to the theories that have been used to define $\mathrm{M}$ (atrix)-theory [8] on $T^{4}$ and $T^{5}[9,10]$. Although the solitonic five-branes in type IIA string theory have the same world-volume theory, describing the theory in terms of Kaluza-Klein monopoles has the special advantage that at a generic point in the moduli space the string coupling can be kept small everywhere, and hence this world volume theory can be described in terms of a weakly coupled string theory. 


\section{Review of Multiple Kaluza-Klein Monopole So- lution}

The multiple Kaluza-Klein monopole solution is described by the metric:

$$
d s^{2}=-d t^{2}+\sum_{m=5}^{D} d y^{m} d y^{m}+d s_{T N}^{2},
$$

where $D$ is 9 for various string theories and 10 for $M$-theory, $y^{m}$ denote the space-like world-volume coordinates on the $(D-4)$-brane represented by this solution, and $d s_{T N}$ is the metric of the Euclidean multi-centered Taub-NUT space [11]:

$$
d s_{T N}^{2}=U^{-1}\left(d x^{4}+\vec{\omega} \cdot d \vec{r}\right)^{2}+U d \vec{r}^{2} .
$$

Here $x^{4}$ denotes the compact direction, and $\vec{r} \equiv\left(x^{1}, x^{2}, x^{3}\right)$ denotes the three spatial coordinates transverse to the brane. $U$ and $\vec{\omega}$ are defined as follows:

$$
U=1+\sum_{I=1}^{N} U_{I}, \quad \vec{\omega}=\sum_{I=1}^{N} \vec{\omega}_{I},
$$

where

$$
U_{I}=\frac{4 m}{\left|\vec{r}-\vec{r}_{I}\right|}
$$

and

$$
\vec{\nabla} \times \vec{\omega}_{I}=\vec{\nabla} U_{I}
$$

$m$ and $\vec{r}_{I}$ are parameters labelling the solution. $\vec{r}_{I}$ can be interpreted as the locations of the Kaluza-Klein monopoles in the transverse space. In order that the solutions be free from conical singularities at $\vec{r}=\vec{r}_{I}, x^{4}$ must have periodicity $16 \pi m$.

The multi-centered Taub-NUT space described by the metric (2.2) supports $N$ linearly independent normalizable self-dual harmonic two forms $\Omega_{I}$, given as follows [3]:

$$
\Omega_{I}=d \xi_{I}
$$

where,

$$
\xi_{I}=U^{-1} U_{I}\left(d x^{4}+\vec{\omega} \cdot d \vec{r}\right)-\vec{\omega}_{I} \cdot d \vec{r} .
$$

Furthermore, as shown in [3], the two-forms defined above satisfy a simple normalization condition:

$$
\int \Omega_{I} \wedge \Omega_{J}=(16 \pi m)^{2} \delta_{I J}
$$




\section{The World-Volume Theory at a Generic Point in the Moduli Space}

Let us now suppose that the original string / M-theory has a $p$-form field $A^{(p)}$. Generalizing the construction of references $[5,7]$ for the case of a single monopole, we can define $N$ different $(p-2)$-form fields $\mathcal{A}_{I}^{(p-2)}$ on the worldvolume through the decomposition:

$$
A^{(p)}\left(\vec{r}, x^{4}, \vec{y}, t\right)=\sum_{I=1}^{N} \mathcal{A}_{I}^{(p-2)}(\vec{y}, t) \wedge \Omega_{I}\left(\vec{r}, x^{4}\right) .
$$

Besides the fields obtained in this way, there are also $3 N$ world-volume scalar fields $\Phi_{I}^{i}(1 \leq i \leq 3)$ associated with the parameters $\vec{r}_{I}$. These can be associated with deformations of the metric following the procedure outlined in reference [3]. This gives the following world-volume field theories of Kaluza-Klein monopoles in different theories at a generic point in the moduli space.

\subsection{M-theory}

This theory contains a three-form gauge field $C_{M N P}$ in (10+1) dimensions. This gives $N$ vector fields on the $(6+1)$ dimensional world volume of the Kaluza-Klein monopole. Together with the $3 N$ scalar fields coming from the translational zero modes, these fields describe the bosonic fields of an $N=1$ supersymmetric $(U(1))^{N}$ gauge theory in $(6+1)$ dimensions. The metric on the moduli space spanned by the scalar fields is determined by the inner product $\int \Omega_{I} \wedge \Omega_{J}$ [3]. From the moduli independence of the inner product given in equation (2.8) we see that this metric is flat, as is expected for this particular supersymmetric field theory $[12]^{2}$.

According to the conjectured duality between M-theory on $S^{1}$ and type IIA string theory, the Kaluza-Klein monopole in M-theory is identified with the Dirichlet six-brane of type IIA string theory. The world-volume dynamics of $N$ well separated Dirichlet six-branes in type IIA string theory is described by an $N=1$ supersymmetric $(U(1))^{N}$ gauge theory in $(6+1)$ dimensions. Thus we see that the dynamics of the Kaluza-Klein monopoles is in agreement with the conjectured duality between M-theory and type IIA string theory.

\footnotetext{
${ }^{2}$ The fermionic fields on the world-volume come from the various fermionic zero modes, but we shall not carry out the explicit analysis here, since the world-volume supersymmetry uniquely fixes the fermionic field content and their interactions.
} 


\subsection{Type IIA string theory}

This theory contains a three-form gauge field $C_{M N P}$ and a one-form field $A_{M}$ from the Ramond-Ramond (RR) sector, and a two-form field $B_{M N}$ from the Neveu-Schwarz-Neveu-Schwarz (NS) sector. $C_{M N P}$ gives $N$ vector fields whereas $B_{M N}$ gives $N$ scalar fields on the $(5+1)$ dimensional world-volume of the monopole. Combining these with the $3 N$ scalar fields coming from the translational zero modes, we get the bosonic field content of $(1,1)$ supersymmetric $(U(1))^{N}$ gauge theory in $(5+1)$ dimensions. Actually, $N$ of these scalar fields, associated with the modes of $B_{M N}$, are periodic coordinates [5]. Thus the world volume field theory is more naturally interpreted as a $(6+1)$ dimensional $N=1$ supersymmetric $(U(1))^{N}$ gauge theory, compactified on a circle of appropriate radius. The $N$ scalar fields originating from $B_{M N}$ are then interpreted as the components of the $(U(1))^{N}$ gauge fields along the compact direction.

In order to see how this result is compatible with the predictions of duality, let us note that first of all, by a T-duality transformation, these Kaluza-Klein monopoles are related to solitonic five-branes of type IIB theory compactified on a circle $S^{1}$, with the five-branes being transverse to $S^{1}$. The S-duality transformation of type IIB string theory converts these solitonic five-branes to Dirichlet five-branes transverse to $S^{1}$. Finally, by a T-duality transformation, this can be mapped to a system of Dirichlet six-branes in type IIA string theory on $S^{1}$ with the six-branes wrapped on the compact direction. For $N$ well separated six-branes, the world-volume theory of this system is given by an $N=1$ supersymmetric $(U(1))^{N}$ gauge theory in $(6+1)$ dimensions, compactified on a circle. Thus we see that the world volume theory of $N$ well separated Kaluza-Klein monopoles in type IIA string theory is consistent with the predictions of duality.

\subsection{Type IIB string theory}

Type IIB string theory contains a four-form field $D_{M N P Q}$ with self-dual field strength, and a pair of two-form gauge fields $B_{M N}$ and $B_{M N}^{\prime}$, one from the NS sector and one from the RR sector. $D_{M N P Q}$ gives rise to $N$ selfdual two-form gauge fields on the (5+1)-dimensional world volume, whereas $B_{M N}$ and $B_{M N}^{\prime}$ give rise to $2 N$ scalar fields on the world volume. Combining these with the $3 N$ scalar fields from the translational zero modes, we get the bosonic field content of $N$ tensor multiplets of the $(2,0)$ supersymmetric field theory in $(5+1)$ dimensions. $2 N$ of the $5 N$ bosonic fields, associated with the modes of $B_{M N}$ and $B_{M N}^{\prime}$, represent periodic coordinates.

In order to see how this is consistent with various duality conjectures, we can make a T-duality transformation to relate it to solitonic five-branes of type IIA on $S^{1}$, with the five branes being transverse to $S^{1}$. By using 
the duality between M-theory on $S^{1}$ and type IIA string theory, we can further relate it to a system of five-branes in M-theory on $T^{2}$, with the five-branes being transverse to both coordinates on $T^{2}$. The world volume theory of this system for $N$ well separated five branes does indeed contain $N$ tensor multiplets of the $(2,0)$ supersymmetry algebra. The $5 N$ bosonic fields represent the five transverse coordinates of each of the $N$ five-branes. Since in this case two of the transverse coordinates are compactified on circles, $2 N$ of the $5 N$ bosonic fields must represent periodic coordinates. Thus we see that the world volume theory of multiple Kaluza-Klein monopoles in type IIB string theory is also consistent with the predictions of duality.

\subsection{Heterotic String Theory}

This theory contains a rank two anti-symmetric tensor field $B_{M N}$, which gives rise to $N$ periodic bosonic fields. These fields, together with the $3 N$ bosonic fields associated with the translational zero modes, constitute the field content of $N$ hypermultiplets of the $(1,0)$ supersymmetry algebra in $(5+1)$ dimensions. However, unlike in the previous cases, in this case the field content, together with the requirement of supersymmetry, does not determine the low energy effective action completely. One needs to specify the moduli space of these hypermultiplets in order to completely describe this theory. According to the result of low energy supergravity theory, this moduli space is flat [3], and is given by $\left(R^{3} \times S^{1}\right)^{N} / S_{N}$, where in the $I$ th term in this product, $R^{3}$ is labelled by the transverse coordinates of the Ith monopole, and $S^{1}$ is labelled by the component of the $B_{M N}$ field along $\Omega_{I}$. $S_{N}$ denotes the effect of symmetrization under permutations of different monopoles. This result however is valid only for large radius $R$ of the compact direction and large separation between the monopoles, since in this case the curvature associated with the solution (2.1), (2.2) is small, and hence higher derivative stringy corrections to the low energy supergravity equations of motion are negligible. However, as we shall now argue, string world-sheet corrections convert this into the moduli space of $N$ BPS monopoles in $S U(2)$ gauge theory [13-15].

To see this, let us recall that a Kaluza-Klein monopole in the heterotic string theory carries 1 unit of Kaluza-Klein monopole charge, and -1 unit of $H$-monopole charge [5]. (The $H$-monopole charge arises from the Lorentz Chern-Simons term in the expression for the field strength of the antisymmetric tensor field.) Now, starting from a situation where the radius of the compact direction is large, and hence the solutions (2.1), (2.2) are valid, consider slowly reducing the radius (keeping the string coupling small) so that it comes close to the self-dual radius. In this case, the low energy effective theory describing heterotic string theory on $S^{1}$ contains a spontaneously broken $S U(2)$ gauge group [16]. This theory will contain BPS 
monopoles associated with this broken $S U(2)$. It is easy to see that this BPS monopole carries precisely the same quantum numbers as the KaluzaKlein monopole. Hence we would expect that as we slowly change the radius from large value to the self-dual point, the moduli space of the Kaluza-Klein monopoles will evolve to that of BPS monopoles. (Note that since we can keep the string coupling small throughout this process, the moduli space approximation never breaks down.)

The question we would like to ask is: what is the metric that interpolates between the moduli space metric of the Kaluza-Klein monopoles and that of BPS monopoles? We shall now show that it is consistent to assume that the correct metric is that of the moduli space of BPS monopoles, and the flat metric on the Kaluza-Klein monopole moduli space emerges from this in an appropriate limit when we ignore higher derivative corrections to the supergravity equations of motion. Implicitly, this may be understood as follows. The moduli space of multiple BPS monopoles involves a scale factor, which, in turn, is determined by the inverse vacuum expectation value (vev) of the Higgs field responsible for the breaking of $S U(2)$ to $U(1)$. If we take the limit where this scale factor goes to zero (i.e. the vev of the Higgs field goes to infinity), keeping the separation between the monopoles fixed, the metric on the moduli space of $N$ BPS monopoles approaches the flat metric. Now, since the Higgs vacuum expectation value in the $S U(2)$ gauge theory is proportional to $\left(R-R^{-1}\right)$ (in units in which $R=1$ denotes the self-dual radius), we see that for large $R$ the Higgs vacuum expectation becomes large, and the scale factor indeed goes to zero. Thus in this limit we expect the moduli space metric of BPS monopoles to approach the flat metric, as we have found for the Kaluza-Klein monopoles.

To see this more explicitly, let us analyse the components of the metric in the Cartesian coordinate system in which the metric for well separated monopoles approaches the identity matrix. In this coordinate system, the various components of the metric on the moduli space of Kaluza-Klein monopoles are naturally functions of $\left(\vec{r}_{I}-\vec{r}_{J}\right) / R \equiv \vec{\xi}_{I J}$ if we restrict ourselves to using the low energy supergravity equations. The Planck scale or the string scale never enters the calculation since Einstein's equations without stringy corrections and without source terms are scale invariant. The result of [3] shows that the metric is the identity matrix, i.e. the metric components do not depend on $\vec{\xi}_{I J}$. On the other hand, components of the metric on the moduli space of BPS monopoles are functions of the combination $\left(R-R^{-1}\right)\left(\vec{r}_{I}-\vec{r}_{J}\right)=\left(R^{2}-1\right) \vec{\xi}_{I J}$ for large $\left(R-R^{-1}\right)\left|\vec{r}_{I}-\vec{r}_{J}\right|$, and these components differ from those of the identity matrix by terms of order $1 /\left(\left(R^{2}-1\right) \xi_{I J}\right)$ when $\left(R^{2}-1\right) \xi_{I J}$ are large [17]. Straightforward dimensional analysis shows us that these terms must be accompanied by the square of the string length in the numerator, and hence are expected to show up in 
the computation of the metric of Kaluza-Klein monopoles only when higher derivative corrections to the supergravity equations of motion are taken into account. In the limit when the string length goes to zero, the metric reduces to the flat metric, as is the case for the moduli space of Kaluza-Klein monopoles in the supergravity theory.

Thus we see that the flat metric on the moduli space of Kaluza-Klein mono-poles as derived from low energy supergravity theory is perfectly consistent with the idea that the correct moduli space is that of multiple BPS monopoles in $S U(2)$ gauge theory, scaled by $\left(R-R^{-1}\right)$. Since the latter moduli space has all the properties required for being consistent with supersymmetry and various other symmetries, and correctly interpolates between the known moduli spaces near the self-dual radius and for large radius of the compact direction, we propose that this is the corrrect moduli space.

\section{Singular Points in the Moduli Space}

Let us now turn to special points in the moduli space where the locations of some of the monopoles coincide. We shall consider the extreme case where the locations of all the $N$ monopoles coincide; any other configuration can be analyzed in an identical manner. This corresponds to taking all the $\vec{r}_{I}$ 's appearing in equation (2.4) to be close to each other. For definiteness, we shall take all of them to be close to $\vec{r}=0$. In this case, the metric near $\vec{r}=0$ can be approximated by:

$$
d s_{T N}^{2} \simeq U^{\prime-1}\left(d x^{4}+\vec{\omega}^{\prime} \cdot d \vec{r}\right)^{2}+U^{\prime} d \vec{r}^{2}
$$

where

$$
U^{\prime}=\sum_{I=1}^{N} \frac{4 m}{\left|\vec{r}-\vec{r}_{N}\right|}, \quad \vec{\nabla} \times \vec{\omega}^{\prime}=\vec{\nabla} U^{\prime} .
$$

But this is precisely the metric on the ALE space $[11,18]$. When all the $\vec{r}_{N}$ 's approach the point $\vec{r}=0$, this space develops an $A_{N-1}$ type singularity, and we get new massless particles / tensionless strings in different string theories / M-theory from branes wrapped around collapsed two-cycles associated with the singularity. We shall now analyze the dynamics of massless particles / tensionless strings in different theories near this singular point and show that in each case the result is in agreement with the predictions of duality. (This analysis is somewhat reminiscent of that in reference [19]). 


\subsection{M-theory}

M-theory on an ALE space with $A_{N-1}$ singularity is expected to have enhanced $S U(N)$ gauge symmetry [20]. Since for well separated monopoles the world volume theory was an $\mathrm{N}=1$ supersymmetric $(U(1))^{N}$ gauge theory in $(6+1)$ dimensions, for coincident monopoles it must be described by an $N=1$ supersymmetric $U(N)$ gauge theory. We had seen earlier that this system is related by duality to a system of $N$ six-branes in type IIA string theory. For coincident six-branes, the world volume theory of this system is indeed described by an $N=1$ supersymmetric $U(N)$ gauge theory. Thus we see that the enhanced gauge symmetry on the world volume of coincident Kaluza-Klein monopoles in M-theory is also consistent with the conjectured duality between M-theory on $S^{1}$ and type IIA string theory.

\subsection{Type IIA String Theory}

Type IIA string theory on an ALE space with $A_{N-1}$ singularity is also expected to have enhanced $S U(N)$ gauge symmetry [20]. Combining this with the result for well separated monopoles, we see that the world-volume theory of $N$ coincident Kaluza-Klein monopoles in type IIA string theory is given by an $N=1$ supersymmetric $U(N)$ gauge theory in $(6+1)$ dimensions compactified on a circle. Our earlier analysis shows that this system is dual to a system of $N$ Dirichlet six branes of type IIA string theory wrapped on a circle. This indeed develops $U(N)$ enhanced gauge symmetry for coincident six branes, showing that the enhancement of gauge symmetry for coincident Kaluza-Klein monopoles is consistent with the duality conjecture.

\subsection{Type IIB String Theory}

Type IIB string theory on an ALE space with $A_{N-1}$ singularity is expected to have no enhanced gauge symmetry, but tensionless self-dual strings [21] whose charges under the $N$ tensor fields lie along the root vectors of $U(N)$. These tensionless strings arise from three-branes wrapped along collapsed two-cycles in the ALE space. To see if this is consistent with duality, recall that the Kaluza-Klein monopoles in type IIB string theory on $S^{1}$ are related by duality to a system of five-branes in M-theory on $T^{2}$, with the fivebranes being transverse to both directions of $T^{2}$. When the-five branes coincide, we do get tensionless strings from membranes stretched between five-branes $[22,23]$. Hence again our result is consistent with the conjectured duality between M-theory on $S^{1}$ and type IIA string theory.

We also have strings of finite tension in the world-volume theory of the five-branes in M-theory on $T^{2}$. These originate from membranes stretched between the five-branes which, instead of following the direct path, wrap once 
or more around the compact directions [10]. One might ask: where do these finite tension strings on the world-volume theory originate in the KaluzaKlein monopoles in type IIB string theory? A straightforward application of duality relations shows us that, in the world-volume of the Kaluza-Klein monopoles in type IIB string theory, these new strings carry the same quantum number as bound states of a three-brane wrapped along a two-cycle, and elementary strings and / or D-strings. Since the electric and magnetic flux of the world-volume gauge field on the three-brane act as sources of $B_{M N}$ and $B_{M N}^{\prime}$ respectively [24,25], we see that in the world volume theory of Kaluza-Klein monopoles in type IIB theory, the new finite tension strings can be identified with three-branes wrapped along collapsed twocycles, together with quantized electric / magnetic flux of the three-brane world-volume gauge field through the two cycle.

\subsection{Heterotic String Theory}

Finally we turn to coincident Kaluza-Klein monopoles in the heterotic string theory. Our earlier assertion that the moduli space of these monopoles is identical to that of multiple BPS monopoles in $S U(2)$ gauge theory implies that there is no singularity in the moduli space even for coincident monopoles, i.e. even when we put the heterotic string theory on a singular ALE space. It will be interesting to find an independent verification of this result.

\section{Conclusion}

In this paper we have studied the dynamics of multiple Kaluza-Klein monopoles in M-theory, and various string theories, for well separated as well as coincident monopoles. In each case, we have found that the dynamics is consistent with all known duality conjectures involving string / M-theory. Dynamics of multiple Kaluza-Klein monopoles in type IIB string theory gives a new realization of the $(2,0)$ supersymmetric theory in $(5+1)$ dimensions with multiple tensor multiplets and tensionless self-dual strings. This theory in appropriate limits might be relevant for describing $\mathrm{M}$ (atrix)-theory on $T^{4}$ and $T^{5}$. We have also argued that the dynamics of $N$ Kaluza-Klein monopoles in heterotic string theory is described by a $(1,0)$ supersymmetric field theory in $(5+1)$ dimensions, with moduli space given by the moduli space of $N$ BPS monopoles in $S U(2)$ gauge theory. 


\section{References}

[1] R. Sorkin, Phys. Rev. Lett. 51 (1983) 87.

[2] D. Gross and M. Perry, Nucl. Phys. B226 (1983) 29.

[3] P. Ruback, Comm. Math. Phys. 107 (1986).

[4] C. Hull, "Gravitational Duality, Branes and Charges", hep-th/9705162.

[5] A. Sen, Phys. Rev. Lett. 79 (1997) 1619.

[6] E. Bergshoeff, B. Janssen and T. Ortin, "Kaluza-Klein Monopoles and Gauged Sigma Models", hep-th/9706117.

[7] Y. Imamura, "Born-Infeld Action and Chern-Simons Term from KaluzaKlein Monopole in M Theory", hep-th/9706144.

[8] T. Banks, W. Fischler, S. Shenker and L. Susskind, Phys. Rev. D55 (1997) 112.

[9] M. Rozali, Phys. Lett. B400 (1997) 260.

[10] M. Berkooz, M. Rozali and N. Seiberg, "Matrix Description of MTheory on $T^{4}$ and $T^{5}$ ", hep-th/9704089.

[11] S. Hawking, Phys. Lett. A60 (1977) 81; G. Gibbons and S. Hawking, Comm. Math. Phys. 66 (1979) 291.

[12] G. Gibbons, G. Papadopoulos and K. Stelle, "HKT and OKT Geometries on Soliton Black Hole Moduli Spaces", hep-th/9706207, and references therein.

[13] N. Manton, Phys. Lett. B110 (1982) 54; Phys. Lett. B154 (1985) 397.

[14] M. Atiyah and N. Hitchin, Phys. Lett. A107 (1985) 21; Phil. Trans. R. Soc. Lond. A315 (1985) 459; "The Geometry and Dynamics of Magnetic Monopoles", Princeton University Press 1988.

[15] G. Gibbons and N. Manton, Nucl. Phys. B274 (1986) 183.

[16] K. Narain, Phys. Lett. B169 (1986) 41; K. Narain, H. Sarmadi and E. Witten, Nucl. Phys. B279 (1987) 369.

[17] G. Gibbons and N. Manton, Phys. Lett. B356 (1995) 32.

[18] T. Eguchi, B. Gilkey and A. Hanson, Phys. Rep. 66 (1980) 213 and references therein. 
[19] M. Bershadsky, V. Sadov and C. Vafa, Nucl. Phys. B463 (1996) 398;

H. Ooguri and C. Vafa, Nucl. Phys. B463 (1996) 55.

[20] E. Witten, Nucl. Phys. B443 (1995) 85.

[21] E. Witten, "Some Comments on String Dynamics", hep-th/9507121.

[22] A. Strominger, Phys. Lett. B383 (1996) 44.

[23] P. Townsend, Phys. Lett. B373 (1996) 68.

[24] E. Witten, Nucl. Phys. B460 (1996) 335.

[25] R. Leigh, Mod. Phys. Lett. A4 (1989) 2767; C. Schmidhuber, Nucl. Phys. B467 (1996) 146; M. Green and M. Gutperle, Phys. Lett. B377 (1996) 28. 